\title{
260. APUNTES SOBRE VARIOS TAXONES GALLEGOS (NW DE LAPENÍNSULA IBÉRICA)
}

\author{
X. Ignacio GONZÁLEZ MARTÍNEZ
}

Recibido el 5 de noviembre de 2014, aceptado para su publicación el 25 de noviembre de 2014

Notes on several Galician taxa (Iberian NW)

Palabras clave. Flora vascular, corología, A Coruña, Galicia, NO ibérico.

Keywords. Vascular flora, chorology, A Coruña, Galicia, Iberian NW.

Como continuación de anteriores aportaciones sobre flora vascular en el SO de la provincia de A Coruña (cf. González-Martínez, 2014a, b), y con el propósito de actualizar en lo posible el conocimiento de la Flora de Galicia, se presentan aquí una serie de aportaciones fruto de diversas prospecciones en distintas unidades de vegetación. En total se incluyen 10 taxones que son novedad para Galicia o para la provincia de A Coruña, así como otros elementos de diverso interés. La mayor parte de las recolecciones fueron realizadas en territorio del "Parque Natural del Complejo Dunar de Corrubedo y Lagunas de Carregal y Vixán" (en adelante, "Parque Natural de Corrubedo"). Esto ha sido debido al trabajo de catalogación de flora vascular que actualmente se está llevando a cabo en dicho espacio. Para cada taxón, ordenados alfabéticamente, se detallan los siguientes datos: municipio, localidad y topónimo, coordenadas UTM del punto de recolección [X, Y (m), 29T ED50], altitud (m.s.n.m.), hábitat, fecha de recolección, autoría y número de pliego del Herbario SANT en el que permanecen depositados los testigos. Además, se hacen diversos comentarios sobre su corología, ecología o características morfológicas.

En cuanto a la nomenclatura botánica, se han utilizado las obras al uso (Flora iberica, Flora Europaea) u otras fuentes actualizadas.
La autoría de los taxones citados en el texto está de acuerdo con las propuestas por Brummitt \& Powell (1992) en sus sucesivas ediciones. Finalmente, para conocer la relevancia de la cita se han consultado diversas floras, trabajos científicos, catálogos y otro tipo de publicaciones, tanto a nivel gallego como nacional. También se ha recurrido al Herbario SANT y al portal electrónico de datos ANTHOS (Sistema de información de las plantas de España, Real Jardín Botánico, www.anthos. es). En las referencias a dicho portal de datos, aparece indicada la fecha de consulta.

Aichryson laxum (Haw.) Bramw.

$=A$. dichotomum (DC.) Webb \& Berthel. $=$ Sempervivum laxum Haw. $=S$. annuum Cut.

ESPAÑA: A Coruña: Ribeira, casco urbano, 501023, 4711448, $6 \mathrm{~m}$, naturalizada en grietas y sobre cubierta briofítica de muros viejos de piedra (Parietarietum judaicae), 10-VI-2014, X. Ignacio González Martínez, SANT 69771.

Se trata de un casmófito suculento endémico de las Islas Canarias, utilizado en jardinería por sus llamativas flores amarillas. En la península ibérica está indicado como subespontáneo para Portugal continental (Ruiz Téllez, 1991: 399; Santos Guerra, 1997: 121, Domingues de Almeida \& Freitas, 2006: 119), en donde aparece formando parte de la comunidad 
rupícola Oryzopsi-Antirrhinetum graniciti. En la presente localidad coloniza de manera abundante muros viejos de piedra, en compañía de la también xenófita Sedum mexicanum Britt. y otras plantas características de la alianza Parietario-Galion muralis.

La presente aportación es novedad para Galicia y, al parecer, para la flora naturalizada de España peninsular.

Baldellia repens (Lam.) Lawalrée subsp. cavanillesii (J.A. Molina, A. Galán, J.M. Pizarro \& Sardinero) Talavera

$=B$. ranunculoides (L.) Parl. subsp. cavanillesi J.A. Molina, A. Galán, J.M. Pizarro \& Sardinero

ESPAÑA: A CORUÑA: Ribeira, Carreira, O Vilar, Parque Natural de Corrubedo, 498040, 4711926, 25 m, interior de sauceda palustre de Salix atrocinerea (Carici-Alnetum glutinosae) y prados higrófilos (Hyperico elodis-Sparganion) temporalmente encharcados, sobre suelo arenoso-limoso de naturaleza higroturbosa, 25-VI-2014, X. Ignacio González Martínez, SANT 69762.

El taxón no aparece citado para la provincia de A Coruña ni en la monografía de Talavera et al. (2008: 313-315) ni en Flora iberica (Talavera et al., 2010: 16). Tampoco se recoge como tal en el Catálogo da Flora de Galicia (Romero-Buján, 2008).

Nuestra aportación constata la presencia de $B$. repens subsp. cavanillesii en la provincia de A Coruña, completándose así la secuencia provincial de Flora iberica (Talavera et al., op . cit.) en lo que respecta a Galicia.

\section{Bergenia crassifolia (L.) Fritsch}

ESPAÑA: A Coruña: Ribeira, Carreira, A Graña, Parque Natural de Corrubedo, en las proximidades de la laguna de Vixán, 498027, 4709668, $23 \mathrm{~m}$, asilvestrada en herbazales nitrófilos sobre suelo arenoso y húmedo, orillando caminos próximos a viviendas, 25-IX-2014, X. Ignacio González Martínez,
SANT 70013.

Se trata de una especie utilizada frecuentemente en jardinería y que parece tener cierta tendencia a escaparse de cultivo y asilvestrarse en ambientes alterados próximos a lugares habitados y vías de comunicación (Rodríguez Romero et al. 2002: 318). Para Galicia encontramos la referencia de Rodríguez Romero (op. cit.), que la citan de Pereiro de Aguiar (Ourense). A la presente localidad es probable que haya llegado a causa de vertidos con restos de jardines.

Novedad provincial para A Coruña y segunda mención para Galicia.

\section{Eucaplyptus pauciflora Sieber ex Spreng. subsp. pauciflora}

ESPAÑA: A Coruña: Ribeira, Carreira, Parque Natural de Corrubedo, al lado las instalaciones de la cantera que se encuentra junto a la carretera O Vilar-Artes, 498269, 4712640, $20 \mathrm{~m}$, eucaliptal con individuos de gran envergadura, sobre suelo húmedo, $X$. Ignacio González Martínez, SANT 69770.

La única referencia gallega que conocemos sobre este eucalipto es la de Silva-Pando et al. (2000: 24), que lo indican para la localidad de Carballedo (Os Peares, Lugo). Nosotros lo encontramos formando grupos homogéneos y también intercalado en plantaciones de $E$. globulus Labill. subsp. globulus.

La cita es novedad provincial.

\section{Linaria arenaria DC.}

ESPAÑA: A Coruña: Ribeira, Aguiño, Couso, "LIC Complejo Húmedo de Corrubedo", 496791, 4708030, c. 4 m, en claros de dunas fijas (Polycarpo-Evacetum ramosissimae), 24-04-2011, X. Ignacio González Martínez, SANT 65309.

Se trata de una planta catalogada "En Peligro Crítico" (CR) según las categorías de la UICN (Bañares et. al. 2004: 382; Moreno, 2008: 59 \& 2010: 24). En el Catálogo Galego de Especies Ameazadas [Decreto 88/2007 
de 19 de abril (DOGA de 9 de mayo); cf. Romero-Buján, 2007] se halla catalogada como especie "En Peligro" (EN).

Su distribución conocida hasta el momento en Galicia está circunscrita a algunos enclaves insulares (Sálvora en la provincia de A Coruña, y Ons, Illa de Arousa y Cíes en la provincia de Pontevedra) y a dos localidades continentales: O Grove (Pontevedra), cuya población cuenta con alrededor de 1000 individuos (Serrano \& Carbajal, com. pers.), y Aguiño (A Coruña) (Merino, 1906: 74; Buch, 1951: 76; Gómez Vigide et al. 1989; Silva-Pando et al. 2000: 27; Serrano \& Carbajal, 2004: 383; Blanco Dios 2008: 323). Silva-Pando et al. (op. cit.) también la indican para Muros (A Coruña), pero en dicha localidad parece haberse extinguido (Lence et al. 2005: 24).

La presente población continental es, hasta el momento, la segunda más importante de Galicia en lo que a número de individuos se refiere (Serrano \& Carbajal, com. pers.). Consta de tres núcleos poblacionales repartidos en un área de 1,36 ha (Tabla 1), todos ellos próximos a la vecina isla de Sálvora, el mayor contingente gallego de la especie y de donde probablemente hayan derivado. En este sentido, estos tres núcleos poblacionales se comportarían como poblaciones locales o "satélites" de un sistema metapoblacional en un modelo "isla-continente", en el que la isla de Sálvora sería la fuente principal de individuos (cf. MacArthur \& Wilson, 1967; Harrison, 1991; Harrison \& Taylor, 1997).

Tras la sospecha de que pudiera haber otros puntos cercanos con presencia de la planta, se prospectaron diferentes áreas adecuadas en el tramo costero comprendido entre O Carreiro (Aguiño; UTM: 499434, 4707256) y la playa de Anquieiro (Carreira; UTM: 497587, 4710251), sin encontrar nuevas poblaciones locales.

A comienzos de junio de 2011 se realizó el censo de esta población continental, siguiendo para ello las indicaciones de Iriondo (2003: 8-14) \& Iriondo et al. (2004: 41, 2009a: 31).
El tamaño total estimado ascendió a 12186 individuos, con densidades relativas que en determinadas parcelas del sector dunar de Area Basta llegaron a los 10 individuos $/ \mathrm{m}^{2}$, semejantes a las obtenidas en la isla de Sálvora (Serrano \& Carbajal op. cit.). En años sucesivos hemos observado significativas oscilaciones demográficas, algo habitual en especies con ciclos anuales, cuyas poblaciones suelen experimentar declives muy acusados y periodos de fuerte crecimiento (Iriondo, 2003:10; López Udias et al. 2009: 167, Iriondo et al. 2009b: 212).

En cuanto a la sinecología de la planta, además de encontrarla en las comunidades ya indicadas por Serrano \& Carbajal (op. cit.) (Polycarpo-Evacetum ramosissimae y Violo-Silenetum littoreae), también la hemos visto creciendo en unidades de OtanthoAmmophiletum australis (Pinisqueira) y Honckenyo-Euphorbietum peplis (As Congreiras).

El grueso de esta población continental (Area Basta), a pesar de hallarse dentro del LIC "Complejo Húmedo de Corrubedo" (ES 1110006), presenta sin embargo graves amenazas y alteraciones. En este sector, la especie muestra una distribución espacial marcadamente heterogénea debido a la fragmentación del hábitat producida por la acción humana. Urge, por tanto, tomar medidas de protección más estrictas que garanticen la conservación de la especie en la zona.

Por último, y dado los cambios poblacionales que esta especie ha experimentado en Galicia en los últimos años (i.e. aumento demográfico en poblaciones conocidas y aparición de nuevas localidades), creemos que la categoría UICN que mejor se ajusta a su actual estatus en la península ibérica es la de "En Peligro" (EN).

\section{Prunus insititia L.}

$=P$. domestica L. subsp. insititia (L.) Bonnier \& Layens

ESPAÑA: A Coruña: Ribeira, Olveira, 


\begin{tabular}{lcccc}
\hline \multicolumn{1}{c}{ Subpoblación } & UTM $(\mathrm{X}, \mathrm{Y})$ & Área $\left(\mathrm{m}^{2}\right)$ & $n$ & Amenazas \\
\hline Area Basta & 496791,4708030 & 13400 & 11727 & $\begin{array}{l}\text { Tránsito de vehículos, pisoteo de } \\
\text { paseantes, extracción de áridos, } \\
\text { entrenamiento de perros de caza }\end{array}$ \\
Pinisqueira & 497364,4707913 & 144 & 450 & Pisoteo de paseantes \\
As Congreiras & 496882,4708758 & 45 & 9 & Pisoteo de paseantes \\
TOTAL & & & 12186 & \\
\hline
\end{tabular}

Tabla 1. Localización geográfica [UTM (m) 29T ED50], superficie de ocupación $\left(\mathrm{m}^{2}\right)$, número de individuos (n) y principales amenazas de las diferentes subpoblaciones. Geographic location [UTM (m) 29T ED50], occupation area $\left(\mathrm{m}^{2}\right)$, number of individuals $(n)$ and main threats of the different subpopulations.

Sirves, Parque Natural de Corrubedo, 497017, 4714913, $7 \mathrm{~m}$, en setos y formando agrupaciones más o menos amplias y cerradas (Prunetalia spinosae), sobre suelo perhúmedo, en compañía de algunos pies de Prunus spinosa y en contacto con prados higrófilos de MolinioArrhenatheretea, 10-VII-2014, X. Ignacio González Martínez, SANT 69757; ibidem, Ribeira, Artes, Parque Natural de Corrubedo, a orillas del río Sanchanás, 497989, 4713960, 8 m, integrado en la aliseda riparia (Senecio-Alnetum glutinosae), en una posición estacionalmente inundada por el desbordamiento del río, 28-VIII-2014, X. Ignacio González Martínez, SANT 69769.

Traemos aquí estos testimonios porque constituyen la segunda mención de esta planta para la provincia de A Coruña, después de que Laínz (1955) la indicase para Santiago [cf. ANTHOS, 08-XI-2014]. No obstante, en el Herbario SANT hemos encontrado otros dos pliegos coruñeses procedentes de Carnota (R.I. Louzán; SANT 37188, 11-06-1994; R.I. Louzán; SANT 37189, 21-06-1994). Otras citas para la mitad occidental de Galicia, en este caso para la provincia de Pontevedra, las encontramos en Laínz (1967) y Amigo et al. (2009: 184, Tabla 1). A pesar de estas referencias, la especie no aparece indicada para dichas provincias ni en el Catálogo da Flora de Galicia (Romero-Buján, 2008: 110) ni en Flora iberica (Blanca \& Díaz de la Guardia, 1998: 450).

Es probable que $P$. insititia se encuentre más extendido en áreas apropiadas del litoral occidental gallego, y que quizás haya pasado desapercibido al haberse asimilado a la variabilidad de P. spinosa L. De éste se diferencia fundamentalmente por presentar frutos y hojas claramente mayores, además de ser constante la pubescencia en pedicelos de flores y frutos (Blanca \& Díaz de la Guardia, op. cit.: 446; Aizpuru et al. 1999: 288; López González, 2010: 527, 535). En la primera localidad hemos observado individuos que por sus características podrían corresponder al híbrido entre estos dos taxones (Prunus $\times$ fruticans Weihe). Muy raramente aparece asilvestrado el afín $P$. domestica L., cultivado por sus frutos y con el podría llegar a confundirse, pero $P$. insititia suele tener espinas y, en el caso de presentarse inerme, posee ramitas jóvenes muy pelosas (López González, op. cit.).

\section{Scilla peruviana $\mathrm{L}$.}

ESPAÑA: A CORUÑA: Ribeira, Carreira, A Graña, Parque Natural de Corrubedo, cerca de la carretera Vixán-A Graña, 498006, 4709663, $25 \mathrm{~m}$, herbazales más o menos ruderalizados, sobre suelo arenoso y con cierta humedad, 
en una zona próxima a viviendas donde también se encuentran las alóctonas Aloe maculata, Bergenia crassifolia, Carpobrotus acinaciformis, Disphyma crassifolium y Watsonia meriana, 29-IV-2014, X. Ignacio González Martínez, SANT 69694.

Aunque autóctona en la península ibérica, su ubicación, la presencia en la zona de varias especies alóctonas y el hecho de tratarse de una planta frecuentemente utilizada como ornamental por sus sus vistosas flores azules, nos hace pensar en un origen antrópico. En esta localidad, encontramos unos 20 individuos repartidos en dos puntos próximos entre sí.

En cualquier caso, el presente testimonio es novedad para la flora gallega.

Scilla verna Huds. subsp. ramburii (Boiss.) K. Richt.

$=S$. ramburii Boiss $=S$. odorata Link ?

ESPAÑA: A CoRUÑA: Ribeira, Carreira, O Vilar, Parque Natural de Corrubedo, al lado de la estación termopluviométrica "Corrubedo", 497783, 4711650, $30 \mathrm{~m}$, pastizal sobre litosuelo arenoso en repisa de roquedo, 30-IV-2014, $X$. Ignacio González Martínez, SANT 69695.

Para la provincia de A Coruña está indicada la presencia de $S$. odorata Link ex Schrader en dos localidades litorales (Ribeira y Porto do Son) (cf. García Martínez et al. 1991: 50), aunque en este trabajo ambas citas no aparecen respaldadas por pliego de herbario. No obstante, dichos testimonios, que además supusieron novedad regional, no aparecen recogidos posteriormente en el Catálogo da Flora de Galicia (Romero-Buján, 2008). Este taxón ha quedado incluido con interrogante dentro de $S$. verna subsp. ramburii según la reciente revisión del grupo $S$. verna Huds. realizada para Flora iberica (Almeida da Silva \& Crespi, 2013: 147-148). En esta monografía, S. verna subsp. ramburii no aparece indicada para A Coruña en la secuencia provincial (Almeida da Silva \& Crespi, op. cit.: 150), aunque las mencionadas referencias coruñesas de $S$. odorata (García
Martínez et al. op. cit.) sí figuran recogidas en la base de datos ANTHOS [08-XI-2014].

En donde la hemos recolectado, aparece de forma muy escasa junto a otras $S$. verna s.l. de controvertida taxonomía (cf. GonzálezMartínez, 2014b: 92), mucho más comunes en el territorio. A diferencia de la subespecie tipo, nuestra planta se caracteriza por presentar una inflorescencia de tipo racemiforme y tener hojas que superan la longitud del escapo (Almeida da Silva \& Crespi op. cit.: 148).

Con esta aportación se reafirma la presencia de esta liliácea en la provincia de A Coruña.

Torilis arvensis (Huds.) Link subsp. purpurea (Ten.) Hayek

ESPAÑA: A CORUÑA: Ribeira, Olveira, Parque Natural de Corrubedo, junto a la pasarela que se dirige a la "Gran Duna", 496421, 4714824, 5 m, pastizal sobre duna fija (Iberidetum procumbentis) con cierta humedad, en una zona con elevado trasiego de paseantes, 10-VII-2014, X. Ignacio González Martínez, SANT 69756.

En el Herbario SANT no hemos encontrado depositado ningún pliego coruñés de esta subespecie, como tampoco menciones a la provincia en el Catálogo da Flora de Galicia (Romero-Buján, 2008), ni en Flora iberica (Jury, 2003: 87). En el Parque Natural de Corrubedo la hemos encontrado únicamente en el punto de recolección y en muy bajo número. Por su ubicación y escasez, quizás se trate de una planta de introducción reciente y fortuita en el territorio.

Novedad para la provincia de A Coruña y por extensión para la mitad occidental de Galicia.

\section{Trifolium ornithopodioides L.}

ESPAÑA: A Coruña: Ribeira, Aguiño, Couso, LIC “Complejo Húmedo de Corrubedo", 497101, 4708389, 19 m, en una explanada utilizada como aparcamiento, sobre suelo húmedo, arenoso y compactado, en unidades 
alteradas de Isoeto-Nanojuncetea, 09-VI-2014, X. Ignacio González Martínez, SANT 69798.

Segunda cita coruñesa de uno de los tréboles gallegos más raros. La primera referencia provincial la encontramos en Valdés Bermejo et al. (1995: 143), que la cita para Louro (Muros). Esta mención pudo servir de base para la indicación provincial entre paréntesis en Flora iberica (Muñoz Rodríguez et al. 2000: 704). Las restantes y escasas citas gallegas corresponden a la provincia de Pontevedra (cf. Merino, 1905: 382; Valdés Bermejo et al. op. cit.).

AGRADECIMIENTOS. A Lola Bermo, Humberto Lens y Cristina Fuentes por su ayuda en los muestreos de Linaria arenaria, y a Miguel Serrano y Roi Carballal, quienes amablemente aportaron información sobre esta planta. Al Dr. Ángel Bañares Baudet por sus precisiones acerca de Aichryson laxum y a Virginia Serrano, que facilitó la localización de la planta. Al Dr. Francisco Javier Silva-Pando por su ayuda en la determinación de Eucalyptus pauciflora subsp. pauciflora. Al personal del Herbario SANT, Ramiro Iglesias, David García-San León y Javier Amigo Vázquez, por su amabilidad, diligencia y amistad. Finalmente, agradecer la labor de un revisor anónimo que contribuyó con diversas precisiones a la mejora de este trabajo.

\section{BIBLIOGRAFÍA}

AIZPURU, I., C. ASEGINOLAZA, P. M. URIBEECHEBARRÍA, P. URRUTIA \& I. ZORRAKIN -1999- Claves ilustradas de la flora del País Vasco y territorios limitrofes. Servicio Central de Publicaciones del Gobierno Vasco, VitoriaGasteiz. 831 pp.

ALMEIDA DA SILVA, R. M. \& A. L. CRESPI -2013- Scilla L. En: S. Castroviejo, E. Rico, M. B. Crespo, A. Quintanar, A. Herrero \& C. Aedo, eds., Flora iberica 20: 145-156. Real Jardín Botánico, CSIC, Madrid.

AMIGO, J., I. PULGAR \& J. IZCO -2009- Evidence of riverside ash tree forest in Southern Galicia (Northwestern Spain). Lazaroa 30: 181-189.

ANTHOS -2014- Sistema de información de las plantas de España. Real Jardín Botánico de
Madrid, CSIC-Fundación Biodiversidad. Recurso electrónico en www.anthos.es.

BAÑARES, Á., G. BLANCA, J. GÜEZMES, J. C. MORENO y S. ORTIZ, eds. -2004- Atlas y Libro Rojo de la Flora Vascular Amenazada de España. Dirección General de Conservación de la Naturaleza, Madrid. 1.069 pp.

BLANCA, G. \& C. DÍAZ DE LA GUARDIA -1998- Prunus L. En: F. Muñoz Garmendia \& C. Navarro, eds., Flora iberica 6: 444-466. Real Jardín Botánico, CSIC, Madrid.

BLANCO-DIOS, J. B. -2008- Notas sobre la flora del Parque Nacional Marítimo-Terrestre de las Islas Atlánticas de Galicia. Acta Bot. Malacitana 33: 322-324.

BRUMMITT, R. K. \& C. E. POWELL -1992Authors of Plant Names. Royal Botanic Gardens Kew, London. 732 pp.

BUCH, H. -1951- Uber die Flora und Vegetation Nordwest-Spaniens. Soc. Scient. Fenn. Comm. Biol. 10(7): 1-98.

DOMINGUES DE ALMEIDA, J. \& H. FREITAS -2006- Exotic naturalized flora of continental Portugal - A reassessment. Bot. Complut. 30: 117-130.

GARCÍA MARTÍNEZ, X.R., E. VALDÉS BERMEJO, F.J. SILVA-PANDO, V. RODRÍGUEZ GRACIA \& F. GÓMEZ VIGIDE -1991- Aportaciones a la Flora de Galicia, IV. Nova Acta Ci. Compostelana (Biol.) 2: 41-56.

GÓMEZ VIGIDE, F., X. R. GARCIA MARTINEZ, E. VALDÉS-BERMEJO, F. J. SILVA-PANDO y V. RODRÍGUEZ GRACIA-1989-Aportaciones a la Flora de Galicia. III. En: F. J. Silva-Pando, ed., Sobre flora y vegetación de Galicia: 101-121. Consellería de Agricultura, Xunta de Galicia. Santiago de Compostela.

GONZÁLEZ-MARTÍNEZ, X. I. -2014a- Nuevos datos sobre xenoflora en el término municipal de Ribeira (A Coruña). Nova Acta Ci. Compostelana (Biol.) 21: 65-76.

GONZÁLEZ-MARTÍNEZ, X. I. -2014b- Nuevos datos sobre flora vascular de Galicia (NO ibérico). Nova Acta Ci. Compostelana (Biol.) 21: 85-97.

HARRISON, S. -1991- Local extinction in a metapopulation context: an empirical evaluation. Biol. J. Linnean Soc. 42: 73-88.

HARRISON, S. \& A. TAYLOR -1997- En: I. Hanski \& M. Gilpin, eds., Empirical evidence 
for metapopulation dynamics: 27-68. Academic Press, London, UK.

IRIONDO, J. M., coord. -2003- Manual de Metodología de Trabajo Corológico y Demográfico para el proyecto Atlas de Flora Amenazada de España. Versión 4.2. 51 pp.

IRIONDO J. M., M. J. ALBERT, Á. BAÑARES, M. DE LA CRUZ, F. DOMÍNGUEZ, A. ESCUDERO, M. B. GARCÍA, D. GUZMÁN, M. MARRERO, J. C. MORENO, H. SAINZ, F. TAPIA \& E. TORRES -2004- Metodología de obtención de datos en las poblaciones naturales. En: Á. Bañares, G. Blanca, J. Güemes, J. C. Moreno y S. Ortiz, eds., Atlas y Libro Rojo de la Flora Vascular Amenazada de España: 39-42. Dirección General de Conservación de la Naturaleza, Madrid.

IRIONDO, J. M., M. J. ALBERT, A. BAÑARES, M. DE LA CRUZ, F. DOMÍNGUEZ LOZANO, A. ESCUDERO, M. B. GARCÍA, D. GUZMÁN, M. MARRERO, J. C. MORENO, H. SAINZ, F. TAPIA \& E. TORRES -2009a- Capítulo II. Metodología. Toma de datos en las poblaciones. En: J. M. Iriondo, M. J. Albert, L. Giménez Benavides, F. Domínguez Lozano \& A. Escudero, eds., Poblaciones en Peligro: Viabilidad Demográfica de la Flora Vascular Amenazada de España: 29-35. Dirección General de Medio Natural y Política Forestal (Ministerio de Medio Ambiente, y Medio Rural y Marino), Madrid.

IRIONDO J. M., M. J. ALBERT, A. ESCUDERO, L. GIMÉNEZ BENAVIDES \& F. DOMÍNGUEZ LOZANO -2009b- Capítulo IV. Análisis general de los resultados. En: J. M. Iriondo, M. J. Albert, L. Giménez Benavides, F. Domínguez Lozano \& A. Escudero, eds., Poblaciones en Peligro: Viabilidad Demográfica de la Flora Vascular Amenazada de España: 207-213. Dirección General de Medio Natural y Política Forestal (Ministerio de Medio Ambiente, y Medio Rural y Marino), Madrid.

JURY, S. L. -2003- Torilis Adans. En: G. Nieto Feliner, S.L. Jury \& A. Herrero, eds., Flora iberica 10: 84-92. Real Jardín Botánico, CSIC, Madrid.

LAÍNZ, M. -1955- Aportaciones al conocimiento de la flora gallega, I. Brotéria, Ci. Nat. 24(51): 108-143.

LAIINZ, M. -1967-Aportaciones al conocimiento de la flora gallega, V. Anales Inst. Forest. Invest.
Exp. 12: 1-51.

LÓPEZ GONZÁLEZ, G. A. -2010- Guía de los árboles y arbustos de la Península Ibérica e Islas Baleares ( $3^{\text {a }}$ Ed. Corr.). Ediciones Mundi-Prensa, Madrid. 894 pp.

LÓPEZ UDIAS, S., C. FABREGAR LLUECA, J. M. IRIONDO, \& M. J. ALBERT -2009Oxytropis jabalambrensis (Pau) Podlech. En: J. M. Iriondo, M. J. Albert, L. Giménez Benavides, F. Domínguez Lozano \& A. Escudero, eds., Poblaciones en Peligro: Viabilidad Demográfica de la Flora Vascular Amenazada de España: 163-167. Dirección General de Medio Natural y Política Forestal (Ministerio de Medio Ambiente, y Medio Rural y Marino), Madrid.

MACARTHUR, R. H. \& E. O. WILSON -1967The theory of island biogeography. Princeton University Press, New Jersey.

MERINO, B. -1905-1906- Flora descriptiva e ilustrada de Galicia I, II. Tipografía Galaica, Santiago de Compostela.

MORENO, J. C., coord. -2008- Lista Roja 2008 de la flora vascular española. Dirección General de Medio Natural y Política Forestal (Ministerio de Medio Ambiente, y Medio Rural y Marino, y Sociedad Española de Biología de la Conservación de Plantas), Madrid. 86 pp.

MORENO, J. C., coord. -2010- Lista Roja 2008 de la flora vascular española. Actualización con los datos del Adenda 2010 al Atlas y Libro Rojo de la Flora Vascular Amenazada. Dirección General de Medio Natural y Política Forestal (Ministerio de Medio Ambiente, y Medio Rural y Marino, y Sociedad Española de Biología de la Conservación de Plantas), Madrid. 46 pp.

MUÑOZ RODRÍGUEZ, A., J. A. DEVESA, \& S. TALAVERA-2000- Trifolium L. En: S. Talavera, C. Aedo, S. Castroviejo, A. Herrero, C. Romero Zarco, F. J. Salgueiro \& M. Velayos, eds., Flora iberica 7(2): 647-719. Real Jardín Botánico, CSIC, Madrid.

RODRÍGUEZ ROMERO, X., A. RODRÍGUEZ ROMERO, A., J. A. DE JESÚS GONZÁLEZ \& V. RODRÍGUEZ GRACIA -2002- Comentarios a la flora de Galicia, X. BAur XXXI: 317-324.

ROMERO-BUJÁN, M. I. -2007- La flora vascular amenazada de Galicia. Catalogación y protección de las especies. Naturalia Cantabricae 3: 15-23.

ROMERO-BUJÁN, M. I. -2008- Catálogo da Flora de Galicia. Monografías do IBADER 
1. Universidade de Santiago de Compostela, Lugo. 172 pp.

RUIZ TÉLLEZ, T. -1991- Contribución al conocimiento de las comunidades de OryzopsiAnthirrinetum granitici corr. Rivas-Martínez 1969, del centro y sur de Portugal, Acta Bot. Malacitana 16(2): 391-403.

SANTOS GUERRA, A. -1997- Aichryson Webb \& Berthel. En: S. Castroviejo, C. Aedo, M. Laínz, R. Morales, F. Muñoz Garmendia, G. Nieto Feliner \& J. Paiva, eds., Flora iberica 5: 119-121. Real Jardín Botánico, CSIC, Madrid.

SERRANO, M. \& CARBAJAL R. -2004- Linaria arenaria DC. En: A. Bañares, G. Blanca, J. Güemes, J. C. Moreno \& S. Ortiz, eds., Atlas y Libro Rojo de la Flora Amenazada de España: 382-383. Dirección General de Conservación de la Naturaleza, Madrid.

SILVA-PANDO, F. J., F. GÓMEZ VIGIDE, X. R. GARCÍA MARTÍNEZ \& J. B. BLANCO-DIOS -2000- Aportacións á flora de Galicia, VI. Nova Acta Ci. Compostelana (Biol.) 10: 21-33.

TALAVERA, S., R. CASIMIRO-SORIEGUER, F. BALAO, J. A. MOLINA \& J. M. PIZARRO -2008- El género Baldellia Parl. (Alismataceae) en la Península Ibérica, Baleares y Marruecos. Acta Bot. Malacitana 33: 309-350.
TALAVERA, S., R. CASIMIRO-SORIEGUER, J.A. MOLINA \& J. M. PIZARRO -2010- Baldellia Parl. En: S. Talavera, M. J. Gallego, C. Romero Zarco \& A. Herreo, eds., Flora iberica 17: 11-18. Real Jardín Botánico, CSIC, Madrid.

VALDÉS BERMEJO, F., F. J. SILVA-PANDO, V. RODRÍGUEZ GRACIA, F. GÓMEZ VIGIDE \& X. R. GARCÍA MARTÍNEZ -1995Aportaciones a la flora de Galicia, V. Nova Acta Ci. Compostelana (Biol.) 5: 135-152.

Dirección del autor. Avda. Miguel Rodríguez Bautista, 23. 15960, Ribeira (A Coruña). xoseignaciogonzalez@yahoo.es 